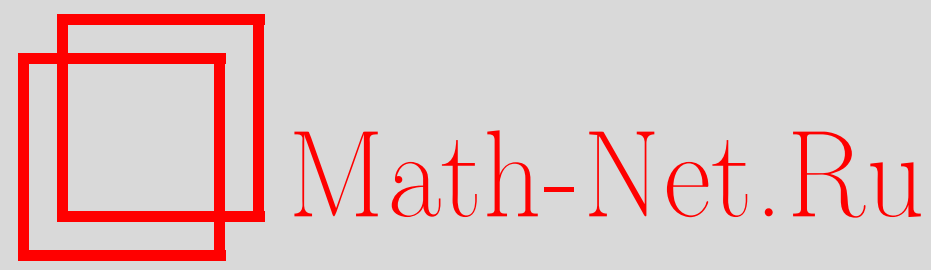

А.-Р. К. Рамазанов, Полиномы, ортогональные со знакочувствительным весом, Матем. заметки, 1996, том 59, выпуск 5, 737-752

DOI: https://doi.org/10.4213/mzm1768

Использование Общероссийского математического портала MathNet.Ru подразумевает, что вы прочитали и согласны с пользовательским соглашением

http://www . mathnet.ru/rus/agreement

Параметры загрузки:

IP : 54.196 .121 .252

26 апреля 2023 г., 14:29:35

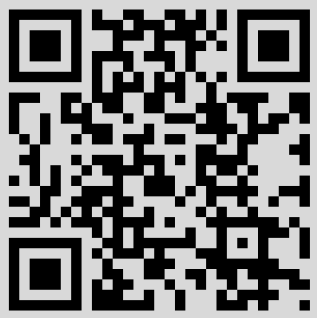


ТОМ 59 вЫПУСК 5 мАЙ 1996

\section{ПОЛИНОМЫ, ОРТОГОНАЛЬНЫЕ СО ЗНАКОЧУВСТВИТЕЛЬНЫМ ВЕСОМ}

\section{А.-P. К. Рамазанов}

Ниже построена система несимметрично ортогональных полиномов, изучены свойства коэффициентов Фурье по этой системе и некоторые общие свойства таких полиномов и, как приложение, приводится квадратурная формула Гаусса.

Рассматриваемая задача возникла под влиянием работ последних лет Е.П. Долженко и Е. А. Севастьянова, в частности, [1] и [2].

\section{§ 1. Существование несимметрично} ортонормированных систем

Следуя [1], упорядоченную пару $\rho(x)=\left(\rho_{-}(x), \rho_{+}(x)\right), x \in(a, b)$, неотрицательных функций $\rho_{-}(x)$ и $\rho_{+}(x)$ назовем знакочувствительным весом на $(a, b)$.

Будем считать, что функции $\rho_{-}(x)$ и $\rho_{+}(x)$ интегрируемы на конечном интервале $(a, b)$, а если $(a, b)$ бесконечен, то существуют конечные интегралы $\int_{a}^{b} x^{m} \rho_{ \pm}(x) d x(m=0,1, \ldots)$, причем в обоих случаях для $m(x, \rho)=$ $\min \left\{\rho_{-}(x), \rho_{+}(x)\right\}, x \in(a, b)$, вьполняется неравенство

$$
\int_{a}^{b} m(x, \rho) d x>0 .
$$

Пусть $c^{+}=\max \{c, 0\}, c^{-}=(-c)^{+}$, и для функции $f(x), x \in(a, b)$, положим

$$
(f, \rho)(x)=f^{+}(x) \rho_{+}(x)-f^{-}(x) \rho_{-}(x) .
$$

Тогда, очевидно, произведение $f(x)(f, \rho)(x) \geqslant 0$.

Класс всех измеримых и почти всюду определенных на $(a, b)$ действительных функций $f(x)$, для которых величина

$$
|f|_{\rho}=|f|_{2, \rho}=\left(\int_{a}^{b} f(x)(f, \rho)(x) d x\right)^{1 / 2}
$$

7 Математические заметки, т. 59 , в. 5

(C) А.-Р. К. РАМАЗАнов 
конечна, обозначим $L_{2, \rho}(a, b)$.

Легко видеть, что при $\rho_{+}(x) \equiv \rho_{-}(x)(x \in(a, b))$ получим класс интегрируемых с квадратом функций (относительно веса $\left.\rho_{+}(x)\right)$.

Отметим также, что в [3] рассмотрено другое обобшение класса интегрируемых с квадратом функций для знакочувствительного веса.

Приведем некоторые свойства функционала $|\cdot| \rho$.

Очевидно, $|f|_{\rho} \geqslant 0 ;|\lambda f|_{\rho}=\lambda|f|_{\rho}$ для неотрицательного числа $\lambda$; вообще говоря, $|-f|_{\rho} \neq|f|_{\rho}$.

Если $f, g \in L_{2, \rho}(a, b)$, то

$$
|f+g|_{\rho} \leqslant|f|_{\rho}+|g|_{\rho}
$$

Действительно, используя, в частности, неравенство Коши-Буняковского, получим

$$
\begin{aligned}
|f+g|_{\rho}^{2} \leqslant & \int_{a}^{b}\left\{\left(f^{+}(x)+g^{+}(x)\right)^{2} \rho_{+}(x)+\left(f^{-}(x)+g^{-}(x)\right) \rho_{-}(x)\right\} d x \\
\leqslant & |f|_{\rho}^{2}+|g|_{\rho}^{2}+2 \int_{a}^{b}\left(f^{+}(x) \sqrt{\rho_{+}(x)}+f^{-}(x) \sqrt{\rho_{-}(x)}\right) \\
& \times\left(g^{+}(x) \sqrt{\rho_{+}(x)}+g^{-}(x) \sqrt{\rho_{-}(x)}\right) d x \leqslant\left(|f|_{\rho}+|g|_{\rho}\right)^{2} .
\end{aligned}
$$

Введем понятие (несимметричного) скалярного произведения. Для $f$, $-f, g \in L_{2, \rho}(a, b)$ скалярным произведением функции $f(x)$ на функцию $g(x)$ относительно знакочувствительного веса $\rho(x)$ назовем величину

$$
(f, g)_{\rho}=\int_{a}^{b} f(x)(g, \rho)(x) d x .
$$

Легко показать, что, вообще говоря, из $f, g \in L_{2, \rho}(a, b)$ не вытекает конечность последнего интеграла.

Однако, этот интеграл существует всегда, когда $f,-f, g \in L_{2, \rho}(a, b)$.

Действительно, имеет место следующий аналог неравенства Коши-Буняковского в $L_{2, \rho}(a, b)$ в этом случае:

$$
\left|(f, g)_{\rho}\right| \leqslant \max \left\{|f|_{\rho},|-f|_{\rho}\right\}|g|_{\rho} .
$$

Для доказательства (3) заметим, что

$$
\begin{aligned}
\left|(f, g)_{\rho}\right| \leqslant \max \left\{\int_{a}^{b}\left[f^{+}(x) g^{+}(x) \rho_{+}(x)+f^{-}(x) g^{-}(x) \rho_{-}(x)\right] d x,\right. & \\
& \left.\int_{a}^{b}\left[f^{-}(x) g^{+}(x) \rho_{+}(x)+f^{+}(x) g^{-}(x) \rho_{-}(x)\right] d x\right\} .
\end{aligned}
$$


Остается оценить каждый из последних интегралов, применив, как и при доказательстве неравенства (2), классическое неравенство Коши-Буняковского для интегралов.

Ясно, что $|f|_{\rho}=\sqrt{(f, f)_{\rho}}$, а при $\rho_{+}(x) \equiv \rho_{-}(x), x \in(a, b)$, величина $(f, g)_{\rho}$ представляет собой обычное скалярное произведение в пространстве функций с интегрируемым квадратом (относительно веса $\rho_{+}(x)$ ).

Если скалярное произведение $(f, g)_{\rho}=0$, то функцию $f \in L_{2, \rho}(a, b)$ назовем ортогональной со знакочувствительным весом $\rho(x)$ (или несимметрично ортогональной с весом $\rho(x))$ к функции $g \in L_{2, \rho}(a, b)$.

В соответствии с этим определением систему функций $\varphi_{0}(x), \varphi_{1}(x), \ldots$ из $L_{2, \rho}(a, b)$ назовем несимметрично ортонормированной или ортонормированной со знакочувствительньм весом $\rho(x)$, если при всех $n, m=0,1, \ldots$ скалярное произведение $\left(\varphi_{n}, \varphi_{m}\right)_{\rho}$ равно нулю при $n>m$ и равно единице при $n=m$.

ТЕорема 1. Если для знакочувствительного веса $\rho(x)=$ $\left(\rho_{-}(x), \rho_{+}(x)\right)$ выполняется условие (1), то существует единственная последовательность алгебрачческих полиномов

$$
P_{0}(x), P_{1}(x), \ldots, P_{n}(x), \ldots,
$$

в которой каждый полином $P_{n}(x)$ имеет степень $n$ и положительный старший коэффициент $a_{n}$ и которая является несимметрично ортонормированной системой с весом $\rho(x)$ на интервале $(a, b)$.

ДокаЗАТЕЛЬСТво. Так как по (1) выполняется

$$
\int_{a}^{b} \rho_{+}(x) d x \geqslant \int_{a}^{b} m(x, \rho) d x>0,
$$

то существует положительная константа $a_{0}$ такая, что

$$
1=\int_{a}^{b} a_{0}^{2} \rho_{+}(x) d x=\int_{a}^{b} a_{0}\left[a_{0}^{+} \rho_{+}(x)-a_{0}^{-} \rho_{-}(x)\right] d x .
$$

Положим $P_{0}(x) \equiv a_{0}$. Тогда легко подобрать $P_{1}(x)=a_{1} x+b_{0}^{(1)} P_{0}(x)$ с $a_{1}>0$ из условий $\left(P_{1}, P_{0}\right)_{\rho}=0,\left(P_{1}, P_{1}\right)_{\rho}=1$.

Допустив, что $P_{0}(x), P_{1}(x), \ldots, P_{n-1}(x)$ уже построены, применим лемму 1.1 из [4] и подберем $P_{n}(x)$ в виде

$$
P_{n}(x)=a_{n} x^{n}+\sum_{k=0}^{n-1} b_{k}^{(n)} P_{k}(x),
$$


используя условия: $a_{n}>0$;

$$
\left(P_{n}, P_{m}\right)_{\rho}=0, \quad m=0,1, \ldots, n-1 ; \quad\left(P_{n}, P_{n}\right)_{\rho}=1 .
$$

Из первого соотношения получим

$$
\begin{gathered}
b_{m}^{(n)}=-a_{n} c_{m}^{(n)} ; \quad c_{m}^{(n)}=\left(x^{n}, P_{m}\right)_{\rho}-\sum_{k=0}^{m-1} c_{k}^{(n)}\left(P_{k}, P_{m}\right)_{\rho} \\
m=1,2, \ldots, n-1 ; \quad c_{0}^{(n)}=\left(x^{n}, P_{0}\right)_{\rho} .
\end{gathered}
$$

Остается найти $a_{n}$, для чего воспользуемся условием $\left(P_{n}, P_{n}\right)_{\rho}=1$. Легко показать, что при

$$
Q(x)=x^{n}-\sum_{k=0}^{n-1} c_{k}^{(n)} P_{k}(x)
$$

имеем

$$
\begin{aligned}
\left(P_{n}, P_{n}\right)_{\rho} & =a_{n}^{2} \int_{a}^{b}\left\{\left[Q^{+}(x)\right]^{2} \rho_{+}(x)+\left[Q^{-}(x)\right]^{2} \rho_{-}(x)\right\} d x \\
& \geqslant a_{n}^{2} \int_{a}^{b}\left\{\left[Q^{+}(x)\right]^{2}+\left[Q^{-}(x)\right]^{2}\right\} m(x, \rho) d x \\
& =a_{n}^{2} \int_{a}^{b} Q^{2}(x) m(x, \rho) d x>0
\end{aligned}
$$

где последнее неравенство вьполняется по лемме 1.2 из [4] (ввиду условия (1)).

Следовательно, $a_{n}>0$ также однозначно определяется.

\section{§2. Коэффициенты Фурье}

Чтобы определить ряд Фурье функции $f \in L_{2, \rho}(a, b)$ по несимметрично ортогональным полиномам (4), потребуем $-f \in L_{2, \rho}(a, b)$. Тогда можно определить коэффициенты Фурье функции $f(x)$ по системе (4) по следующим формулам:

$$
\begin{aligned}
& a_{0}=a_{0}(f)=\left(f, P_{0}\right)_{\rho}, \\
& a_{n}=a_{n}(f)=\left(f, P_{n}\right)_{\rho}-\sum_{k=0}^{n-1} a_{k}(f)\left(P_{k}, P_{n}\right)_{\rho}, \quad n=1,2, \ldots
\end{aligned}
$$

Заметим, что $-f \in L_{2, \rho}(a, b)$ является, вообще говоря, необходимьм условием существования коэффищиентов Фурье (5) для произвольно взятой функции $f \in L_{2, \rho}(a, b)$. 
Действительно, пусть $f(x)=-1 / \sqrt{x}, \rho_{+}(x)=1 / \sqrt{x}, \rho_{-}(x)=x$, $x \in(0,1)$. Тогда, очевидно, $f \in L_{2, \rho}(0,1)$, но коэффициент $a_{0}(f)$ неопределен.

Рассмотрим ряд Фурье по полиномам (4) и его частные суммы:

$$
\begin{gathered}
f(x) \sim \sum_{n=0}^{\infty} a_{n}(f) P_{n}(x), \\
S_{n}(x)=S_{n}(x, f)=\sum_{k=0}^{n} a_{k}(f) P_{k}(x), \quad n=0,1, \ldots
\end{gathered}
$$

Тогда из (5) для $n=1,2, \ldots$ следует, что

$$
a_{n}(f)=\left(f-S_{n-1}, P_{n}\right)_{\rho} .
$$

Отметим некоторые свойства коэффициентов Фурье (5).

Если $c_{0}, c_{1}, \ldots, c_{n}$ - коэффишиенты разложения произвольного алгебраического полинома $P(x)$ степени $n(n=0,1, \ldots)$ по полиномам системы (4) $\left(c_{k}-\right.$ коэффициенты при $\left.P_{k}(x)\right)$, то легко подсчитать, что $c_{k}=a_{k}(P), k=0,1, \ldots, n$.

Более того, если частные суммы $R_{n}(x)$ ряда вида $\sum_{k=0}^{\infty} c_{k} P_{k}(x)$ сходятся к некоторой функции $f \in L_{2, \rho}(a, b)$ по "норме" $|\cdot|_{\rho}$, как при $\rho(x)=$ $\left(\rho_{-}(x), \rho_{+}(x)\right)$, так и при $\rho(x)=\left(\rho_{+}(x), \rho_{-}(x)\right)$, то необходимо $c_{k}=a_{k}(f)$ для каждого $k=0,1, \ldots$

Действительно, равенство $c_{0}=a_{0}(f)$ получается, если применить (3) к правой части равенства

$$
\left|a_{0}(f)-c_{0}\right|=\left|\int_{a}^{b}\left[f(x)-R_{n}(x)\right]\left(P_{0}, \rho\right)(x) d x\right| .
$$

Пусть уже показано $c_{m}=a_{m}(f)$ при $m=0,1, \ldots, k-1$ и пусть $n>k$. Тогда, как и вьше, требуемое получается из равенств

$$
\begin{aligned}
\int_{a}^{b}\left[f(x)-R_{n}(x)\right] & \left(P_{k}, \rho\right)(x) d x \\
= & \left(f, P_{k}\right)_{\rho}-\sum_{m=0}^{k-1} c_{m}\left(P_{m}, P_{k}\right)_{\rho}-c_{k}=a_{k}(f)-c_{k}
\end{aligned}
$$

Аналогичное утверждение имеет место и в случае равномерно сходяшихся рядов по системе (4).

Отметим также следующее легко проверяемое свойство. 
Пусть $f,-f \in L_{2, \rho}(a, b)$. Для того чтобы $a_{0}, a_{1}, \ldots, a_{n}$ были коэффициентами Фурье функции $f(x)$ по системе (4), необходимо и достаточно выполнение при $m=0,1, \ldots, n$ равенства

$$
\int_{a}^{b}\left[f(x)-\sum_{k=0}^{n} a_{k} P_{k}(x)\right]\left(P_{m}, \rho\right)(x) d x=0 .
$$

Для получения аналогов тождества Бесселя и равенства Парсеваля наряду с коэффициентами Фурье $a_{n}=a_{n}(f)$, определяемыми по формулам (5), рассмотрим также следуюшие:

$$
b_{n}=b_{n}(f)=\int_{a}^{b}(f, \rho)(x) P_{n}(x) d x, \quad n=0,1, \ldots .
$$

Тогда легко проверить, что для любого набора чисел $c_{0}, c_{1}, \ldots, c_{n}$ выполняется равенство

$$
\begin{aligned}
\int_{a}^{b}\left[f(x)-S_{n}(x)\right]\left[(f, \rho)(x)-\sum_{k=0}^{n} c_{k}\left(P_{k}, \rho\right)(x)\right] d x \\
=\int_{a}^{b} f(x)(f, \rho)(x) d x-\sum_{k=0}^{n} a_{k} b_{k}
\end{aligned}
$$

или, учитьвая свойство (8) коэффициентов $a_{k}=a_{k}(f)$, имеем

$$
\int_{a}^{b}\left[f(x)-S_{n}(x)\right](f, \rho)(x) d x=\int_{a}^{b} f(x)(f, \rho)(x) d x-\sum_{k=0}^{n} a_{k} b_{k} .
$$

Следовательно, стремление для данных функции $f(x)$ и веса $\rho(x)$ интеграла в левой части $(9)$ к нулю (при $n \rightarrow \infty)$ необходимо и достаточно для вьполнения следуюшего аналога равенства Парсеваля:

$$
\int_{a}^{b} f(x)(f, \rho)(x) d x=\sum_{n=0}^{\infty} a_{n} b_{n}
$$

Заметим, что для $f,-f, g \in L_{2, \rho}(a, b)$ вполне аналогично (9) имеем также равенство

$$
\begin{aligned}
& \int_{a}^{b}\left[f(x)-S_{n}(x, f)\right](g, \rho)(x) d x \\
&=\int_{a}^{b} f(x)(g, \rho)(x) d x-\sum_{k=0}^{n} a_{k}(f) b_{k}(g) .
\end{aligned}
$$


Желая получить аналог теоремы $\Phi$. Рисса-Э. Фишера, рассмотрим линейное пространство $L_{2, \rho}^{*}(a, b)$ всех измеримых функций $f(x), x \in(a, b)$, для которых существует конечньй интеграл

$$
\int_{a}^{b} f(x)(g, \rho)(x) d x, \quad g \in L_{2, \rho}(a, b)
$$

при этом функции $f(x)$, для которых указанньй интеграл равен нулю при всех $g \in L_{2, \rho}(a, b)$, считаем нулевым элементом $L_{2, \rho}^{*}(a, b)$.

Для $f \in L_{2, \rho}^{*}(a, b)$ введем норму, полагая

$$
\|f\|_{\rho}=\sup \left|\int_{a}^{b} f(x)(g, \rho)(x) d x\right|,
$$

где супремум берется по всем $g \in L_{2, \rho}(a, b)$ таким, что $|g|_{\rho} \leqslant 1$.

Покажем, что $\|f\|_{\rho}<\infty$ для любой $f \in L_{2, \rho}^{*}(a, b)$.

Допустим, что $\|f\|_{\rho}=\infty$ для некоторой такой функции. Тогда, очевидно, вьполняется хотя бы одно из равенств

$$
\sup \int_{a}^{b}|f(x)| g^{ \pm}(x) \rho_{ \pm}(x) d x=\infty,
$$

где супремумы берутся по всем $g \in L_{2, \rho}(a, b)$ с $|g|_{\rho} \leqslant 1$.

Пусть, например, выполняется равенство со знаком ' + '. Тогда существует последовательность $g_{k} \in L_{2, \rho}(a, b)$ с $\left|g_{k}\right|_{\rho} \leqslant 1$ такая, что

$$
\int_{a}^{b}|f(x)| g_{k}^{+}(x) \rho_{+}(x) d x \geqslant 2^{k}, \quad k=1,2, \ldots
$$

Рассмотрим неотрицательные функции

$$
G_{n}(x)=\sum_{k=1}^{n} 2^{-k} g_{k}^{+}(x), \quad n=1,2, \ldots
$$

Тогда, очевидно,

$$
\left|G_{n}\right|_{\rho}=\int_{a}^{b} G_{n}^{2}(x) \rho_{+}(x) d x<1 .
$$

$\mathrm{K}$ возрастающей последовательности функций $G_{n}^{2}(x) \rho_{+}(x)(n=1$, $2, \ldots)$ и функции $g^{2}(x) \rho_{+}(x)$, где $g(x)=\sum_{k=1}^{\infty} 2^{-k} g_{k}^{+}(x)$, применим теорему Б. Леви о предельном переходе в интегралах, что дает $|g|_{\rho} \leqslant 1$.

С другой стороны, при $n=1,2, \ldots$ имеем

$$
\int_{a}^{b}|f(x)| G_{n}^{+}(x) \rho_{+}(x) d x \geqslant n,
$$


и снова по теореме Б. Леви $\int_{a}^{b}|f(x)| g(x) \rho_{+}(x) d x=\infty$, что противоречит $f \in L_{2, \rho}^{*}(a, b)$.

Следующая лемма устанавливает связь между $|f|_{\rho},\|f\|_{\rho}$ и величиной

$$
\|\left. f\right|_{\rho}=\sup \int_{a}^{b}|f(x)(g, \rho)(x)| d x
$$

где супремум берется по всем $g \in L_{2, \rho}(a, b)$ с $|g|_{\rho} \leqslant 1$.

ЛЕмма. Если $\pm f \in L_{2, \rho}(a, b)$, mо $f \in L_{2, \rho}^{*}(a, b)$ и вилолняются соотношения

$$
\begin{aligned}
& \|f\|_{\rho}=\max \left\{|f|_{\rho},|-f|_{\rho}\right\}, \\
& \|f\|_{\rho} \leqslant\left\|\left.f\right|_{\rho} \leqslant|f|_{\rho}+|-f|_{\rho} \leqslant 2\right\| f \|_{\rho} .
\end{aligned}
$$

ДокАЗАТЕЛЬСтво. Первая часть леммы и неравенство

$$
\|f\|_{\rho} \leqslant \max \left\{|f|_{\rho},|-f|_{\rho}\right\}
$$

непосредственно следуют из (3). Пусть теперь $|f|_{\rho} \neq 0$. Тогда $\left.|f /| f\right|_{\rho} \mid=1$. Поэтому

$$
\|f\|_{\rho} \geqslant \int_{a}^{b} f(x)\left(\frac{f}{|f|_{\rho}}, \rho\right)(x) d x=|f|_{\rho} .
$$

Аналогично, $|-f|_{\rho} \leqslant\|-f\|_{\rho}=\|f\|_{\rho}$.

Случаи $|f|_{\rho}=0$ и $|-f|_{\rho}=0$ очевидны, а поэтому требуемое в лемме равенство доказано.

Во втором соотношении крайние неравенства очевидны, докажем средние. Для $\pm f, g \in L_{2, \rho}(a, b)$ имеем

$$
\begin{aligned}
\int_{a}^{b}|f(x)(g, \rho)(x)| d x \leqslant & \int_{a}^{b}\left|f^{+}(x) g^{+}(x) \rho_{+}(x)+f^{-}(x) g^{-}(x) \rho_{-}(x)\right| d x \\
& +\int_{a}^{b}\left|f^{+}(x) g^{-}(x) \rho_{-}(x)+f^{-}(x) g^{+}(x) \rho_{+}(x)\right| d x \\
\leqslant & \int_{a}^{b}\left(f^{+}(x) \sqrt{\rho_{+}(x)}+f^{-}(x) \sqrt{\rho_{-}(x)}\right) \\
& \times\left(g^{+}(x) \sqrt{\rho_{+}(x)}+g^{-}(x) \sqrt{\rho_{-}(x)}\right) d x \\
& +\int_{a}^{b}\left(f^{-}(x) \sqrt{\rho_{+}(x)}+f^{+}(x) \sqrt{\rho_{-}(x)}\right) \\
\times & \left(g^{+}(x) \sqrt{\rho_{+}(x)}+g^{-}(x) \sqrt{\rho_{-}(x)}\right) d x .
\end{aligned}
$$

Остается воспользоваться классическим неравенством Коши-Буняковского для интегралов и перейти к супремумам при $|g|_{\rho} \leqslant 1$. 
Пусть, как и выше, $a_{k}(f)$ и $b_{k}(g)(k=0,1, \ldots)$ определяются для $f \in$ $L_{2, \rho}^{*}(a, b)$ и $g \in L_{2, \rho}(a, b)$ по равенствам $(5)$ и $\left(5^{\prime}\right)$. Так как для полиномов из системы (4) выполняется равенство $\left|P_{k}\right|_{\rho}=1$ при $k=0,1, \ldots$, то коэффициенты Фурье $a_{k}(f)(k=0,1, \ldots)$ определены для любой функции $f \in L_{2, \rho}^{*}(a, b)$.

Ниже вес $\rho(x)=\left(\rho_{-}(x), \rho_{+}(x)\right), x \in(a, b)$, назовем стандартньг, если для любой $f \in L_{2, \rho}^{*}(a, b)$ имеем

$$
\lim _{n \rightarrow \infty} \sup \sum_{k=n}^{\infty} a_{k}(f) b_{k}(g)=0,
$$

где супремум берется по всем $g \in L_{2, \rho}(a, b)$ с $|g|_{\rho} \leqslant 1$.

Заметим, что при $\rho_{+}(x) \equiv \rho_{-}(x), x \in(a, b)$, вес $\rho(x)$ является стандартным. В этом случае система (4) представляет собой ортонормированную систему полиномов с весом $\rho_{+}(x), x \in(a, b)$, причем для $k=0,1, \ldots$ имеем

$$
a_{k}:=a_{k}(f)=\int_{a}^{b} f(x) P_{k}(x) \rho_{+}(x) d x, \quad b_{k}:=b_{k}(g)=a_{k}(g) .
$$

Поэтому, применив неравенство Коши-Буняковского для рядов, имеем

$$
\left|\sum_{k=n}^{\infty} a_{k} b_{k}\right| \leqslant\left(\sum_{k=n}^{\infty} a_{k}^{2}\right)^{1 / 2}\left(\sum_{k=n}^{\infty} b_{k}^{2}\right)^{1 / 2} .
$$

Остается воспользоваться имеющими место в этом случае сходимостью ряда $a_{0}^{2}+a_{1}^{2}+\cdots$ и неравенством Бесселя:

$$
\sum_{k=n}^{\infty} b_{k}^{2} \leqslant\left(\int_{a}^{b} g^{2}(x) \rho_{+}(x) d x\right)^{1 / 2}=|g|_{\rho} \leqslant 1 .
$$

Теорема 2. Пусть $P_{0}(x), P_{1}(x), \ldots$ - несимметрично ортонормированная система со знакочувствительным весом $\rho(x)=\left(\rho_{-}(x)\right.$, $\left.\rho_{+}(x)\right), x \in(a, b)$. Если для числовой последовательности $c_{0}, c_{1}, \ldots$ выполняется соотношение

$$
\lim _{n \rightarrow \infty} \sup \left|\sum_{k=n}^{\infty} c_{k} b_{k}(g)\right|=0,
$$

где супремум берется по всем $g \in L_{2, \rho}(a, b)$ c $|g|_{\rho} \leqslant 1$, то существует функиия $f \in L_{2, \rho}^{*}(a, b)$ такая, что $a_{k}(f)=c_{k}(k=0,1, \ldots)$, причем

$$
\int_{a}^{b} f(x)(g, \rho)(x) d x=\sum_{k=0}^{\infty} a_{k}(f) b_{k}(g)
$$

для любой $g \in L_{2, \rho}(a, b)$. 
ДоКАЗАТЕЛЬСТВо. ПолоЖим $S_{n}(x)=\sum_{k=0}^{n} c_{k} P_{k}(x), n=0,1, \ldots$ Тогда для натуральных $n \geqslant m$ и любой $g \in L_{2, \rho}(a, b)$ c $|g|_{\rho} \leqslant 1$ имеем

$$
\int_{a}^{b}\left(S_{n}(x)-S_{m}(x)\right)(g, \rho)(x) d x=\sum_{k=m+1}^{n} c_{k} b_{k}(g) .
$$

Отсюда и из условия теоремы легко следует, что $\left\|S_{n}-S_{m}\right\|_{\rho} \rightarrow 0$ при $n, m \rightarrow \infty$, и по лемме ||$S_{n}-\left.S_{m}\right|_{\rho} \rightarrow 0$ при $n, m \rightarrow \infty$.

Следовательно, для любой $g \in L_{2, \rho}(a, b)$ с $|g|_{\rho} \leqslant 1$ получим

$$
\int_{a}^{b}\left|\left(S_{n}(x)-S_{m}(x)\right)(g, \rho)(x)\right| d x \rightarrow 0 \quad(n, m \rightarrow \infty) .
$$

Теперь по аналогии с доказательством полноты пространства суммируемых функций (см., например, [6]) показьваем, что сушествует $f \in L_{2, \rho}^{*}(a, b)$, для которой $\| S_{n}-\left.f\right|_{\rho} \rightarrow 0$ при $n \rightarrow \infty$, а следовательно, по лемме $\left\|S_{n}-f\right\|_{\rho} \rightarrow 0$ при $n \rightarrow \infty$.

Остается показать равенства $a_{k}(f)=c_{k}, k=0,1, \ldots$, что непосредственно следует из неравенства

$$
\left|a_{k}(f)-c_{k}\right| \leqslant\left\|f-S_{n}\right\|_{\rho} \quad(n>k),
$$

которое легко получить по индукции, используя формулы (5) и равенства $\left|P_{k}\right|_{\rho}=1, k=0,1, \ldots$.

Итак, доказано, что $S_{n}(x)=S_{n}(x, f), n=0,1, \ldots$ Тогда, взяв для любой $g \in L_{2, \rho}(a, b)$ с $|g|_{\rho}>0$ (случай $|g|_{\rho}=0$ очевиден) функцию $G(x)=g(x) /|g|_{\rho}$, легко показать, что

$$
\begin{aligned}
& \int_{a}^{b} f(x)(G, \rho)(x) d x-\sum_{k=0}^{n} a_{k}(f) b_{k}(G) \\
&=\int_{a}^{b}\left[f(x)-S_{n}(x, f)\right](G, \rho)(x) d x, \quad n=0,1, \ldots
\end{aligned}
$$

Остается перейти к модулям обеих частей этого равенства, затем в правой части взять супремум по всем $G \in L_{2, \rho}(a, b)$ с $|G|_{\rho} \leqslant 1$ и учесть $\left\|f-S_{n}\right\|_{\rho} \rightarrow 0(n \rightarrow \infty)$.

Наконец, в левой части заменив $G(x)$ на $g(x) /|g|_{\rho}$, получим требуемое в теореме равенство.

Теорема 2 доказана.

Заметим, что существует только одна функция в $L_{2, \rho}^{*}(a, b)$, удовлетворяюшая обоим условиям теоремы 2.

Действительно, для двух таких функций $f_{1}(x)$ и $f_{2}(x)$ имели бы $a_{k}\left(f_{1}\right)=a_{k}\left(f_{2}\right)(k=0,1, \ldots)$, а поэтому из аналога равенства Парсеваля для любой $g \in L_{2, \rho}(a, b)$ получили бы

$$
\int_{a}^{b}\left[f_{1}(x)-f_{2}(x)\right](g, \rho)(x) d x=0 .
$$




\section{§ 3. О полноте и замкнутости системы}

Пусть $P_{0}(x), P_{1}(x), \ldots$ является несимметрично ортонормированной системой с весом $\rho(x)=\left(\rho_{-}(x), \rho_{+}(x)\right), x \in(a, b)$.

Эту систему назовем замкнутой относительно $L_{2, \rho}^{*}(a, b)$, если для любых $f \in L_{2, \rho}^{*}(a, b)$ и $g \in L_{2, \rho}(a, b)$ вьполняется равенство

$$
\int_{a}^{b} f(x)(g, \rho)(x) d x=\sum_{k=0}^{\infty} a_{k}(f) b_{k}(g),
$$

где $a_{k}(f)$ и $b_{k}(g)$ определяются по формулам $(5)$ и $\left(5^{\prime}\right)$.

Систему $P_{0}(x), P_{1}(x), \ldots$ назовем полной относительно $L_{2, \rho}^{*}(a, b)$, если его нулевым элементом является любая $\varphi \in L_{2, \rho}^{*}(a, b)$, для которой имеет место хотя бы одно из следующих двух условий:

i) $\int_{a}^{b} \varphi(x)\left(P_{k}, \rho\right)(x) d x=0$ при всех $k=0,1, \ldots$;

ii) $\pm \varphi \in L_{2, \rho}(a, b)$ и при некотором целом неотрицательном $n$ предыдущее равенство выполняется для $k=0,1, \ldots, n-1$, а для $k=n, n+1, \ldots$ выполняются равенства

$$
\int_{a}^{b} P_{k}(x)( \pm \varphi, \rho)(x) d x=0 .
$$

Отметим некоторые свойства полных и замкнутьх относительно $L_{2, \rho}^{*}(a, b)$ систем.

I. Чтобы для любой функции $f \in L_{2, \rho}^{*}(a, b)$ ряд Фурье сходился к ней по норме $L_{2, \rho}^{*}(a, b)$, необходимо и достаточно вьполнение одновременно двух условий:

а) вес $\rho(x)$ является стандартньм;

б) система $P_{0}(x), P_{1}(x), \ldots$ является замкнутой.

Это свойство легко следует из аналога тождества Бесселя $\left(9^{\prime}\right)$.

II. Если система $P_{0}(x), P_{1}(x), \ldots$ замкнутая, то она является полной.

Действительно, если для $\varphi \in L_{2, \rho}^{*}(a, b)$ выполнено условие і) из определения полной системы, то по индукции можно показать, что все коэффициенты $\Phi$ урье $a_{k}(\varphi)=0, k=0,1, \ldots$ Остается для $\varphi(x)$ и произвольной $g \in L_{2, \rho}(a, b)$ воспользоваться определением замкнутой системы.

Если же выполнено условие іi), то при некотором целом неотрицательном $n$ имеем $a_{k}(\varphi)=0$ для $k=0,1, \ldots, n-1$ и $b_{k}(\varphi)=0$ для $k=n, n+1, \ldots$.

Отсюда, из определения замкнутой системы и $\varphi \in L_{2, \rho}(a, b)$ получим

$$
|\varphi|_{\rho}^{2}=\int_{a}^{b} \varphi(x)(\varphi, \rho)(x) d x=\sum_{k=0}^{\infty} a_{k}(\varphi) b_{k}(\varphi)=0 .
$$


Аналогично, $|-\varphi|_{\rho}=0$.

По лемме из $\S 2\|\varphi\|_{\rho}=0$, откуда легко следует, что $\varphi(x)$ нулевой элемент $L_{2, \rho}^{*}(a, b)$.

III. Если система $P_{0}(x), P_{1}(x), \ldots$ со стандартным весом $\rho(x)$ полная, то она является замкнутой.

Допустим, что (ср. [6, с. 170]) существуют $f \in L_{2, \rho}^{*}(a, b)$ и $G \in L_{2, \rho}(a, b)$, для которых

$$
\int_{a}^{b} f(x)(G, \rho)(x) d x \neq \sum_{k=0}^{\infty} a_{k}(f) b_{k}(G) .
$$

Так как вес стандартньй, то коэффициенты $a_{k}(f), k=0,1, \ldots$, удовлетворяют условию теоремы 2 , по которой существует такая функция $F \in L_{2, \rho}^{*}(a, b)$, что $a_{k}(F)=a_{k}(f)(k=0,1, \ldots)$ и, в частности,

$$
\int_{a}^{b} F(x)(G, \rho)(x) d x=\sum_{k=0}^{\infty} a_{k}(f) b_{k}(G) .
$$

Из $a_{k}(F-f)=0(k=0,1, \ldots)$, применив индукцию, для $k=0,1, \ldots$ получим

$$
\int_{a}^{b}(F(x)-f(x))\left(P_{k}, \rho\right)(x) d x=0,
$$

и в силу полноты системы для любой $g \in L_{2, \rho}(a, b)$ имеем

$$
\int_{a}^{b}(F(x)-f(x))(g, \rho)(x) d x=0
$$

что, очевидно, невозможно при $g(x)=G(x)$.

IV. Если система $P_{0}(x), P_{1}(x), \ldots$ со стандартным весом $\rho(x)$ полная, то для любой функции $f \in L_{2, \rho}^{*}(a, b)$ ее коэффициенты Фурье $a_{k}(f) \rightarrow 0$ при $k \rightarrow \infty$.

Действительно, из формул (5) для $n=1,2, \ldots$ следует, что $a_{n}(f)=$ $\left(f-S_{n-1}, P_{n}\right)_{\rho}$, где $S_{n-1}(x)=S_{n-1}(x, f)$. Отсюда, так как $\left|P_{n}\right|_{\rho}=1$, имеем $\left|a_{n}(f)\right| \leqslant\left\|f-S_{n-1}\right\|_{\rho}$. Остается воспользоваться свойствами III и I.

\section{$\S 4$. О сопряженной несимметрично}

\section{ортонормированной системе полиномов}

Систему функций $\varphi_{0}(x), \varphi_{1}(x), \ldots$ из $L_{2, \rho}(a, b)$ назовем сопряженной несимметрично ортонормированной системой со знакочувствительным весом $\rho(x)=\left(\rho_{-}(x), \rho_{+}(x)\right), x \in(a, b)$, если $\left(\varphi_{n}, \varphi_{m}\right)_{\rho}=0$ при $n<m$ и $\left(\varphi_{n}, \varphi_{n}\right)_{\rho}=1(n, m=0,1, \ldots)$. 
Теорема $1^{\prime}$. Если для $\rho(x)=\left(\rho_{-}(x), \rho_{+}(x)\right), x \in(a, b)$, выполняется условие (1), то существует единственная последовательность алгебраических полиномов

$$
Q_{0}(x), Q_{1}(x), \ldots, Q_{n}(x), \ldots
$$

с положительными стариими коэффициентами, $\operatorname{deg} Q_{n}=n(n=0$, $1, \ldots)$, которая образует сопряженную несимметрично ортонормированную систему с весом $\rho(x)$ на $(a, b)$.

ДокАЗАТЕЛЬСТво аналогично доказательству теоремы 1 , причем $Q_{n}(x)(n=1,2, \ldots)$ следует выбрать в виде

$$
Q_{n}(x)=a_{n}\left(x^{n}-b_{0} Q_{0}(x)-b_{1} Q_{1}(x)-\ldots-b_{n-1} Q_{n-1}(x)\right),
$$

используя условия: $a_{n}>0$;

$$
\left(Q_{k}, Q_{n}\right)_{\rho}=0, \quad k=0,1, \ldots, n-1 ; \quad\left(Q_{n}, Q_{n}\right)_{\rho}=1 .
$$

Пусть

$$
\begin{gathered}
E_{1}=\left\{x \in(a, b) \mid Q_{n}(x) \geqslant 0\right\}, \quad E_{2}=(a, b) \backslash E_{1} \\
a_{k m}=\int_{E_{1}} Q_{k}(x) Q_{m}(x) \rho_{+}(x) d x+\int_{E_{2}} Q_{k}(x) Q_{m}(x) \rho_{-}(x) d x, \\
k, m=0,1, \ldots, n-1 .
\end{gathered}
$$

Для определения $b_{0}, b_{1}, \ldots, b_{n-1}$ получится система линейных алгебраических уравнений, у которой при вьполнении условия (1) главньй определитель (аналог определителя Грама)

$$
\operatorname{det}\left[a_{k m}\right]_{k, m=0}^{n-1} \neq 0 .
$$

Действительно, в противном случае легко показать, что сушествует нетривиальньй набор чисел $b_{0}, b_{1}, \ldots, b_{n-1}$, для которого

$$
\sum_{k=0}^{n-1} \sum_{m=0}^{n-1} a_{k m} b_{k} b_{m}=0,
$$

чего не может быть, так как при выполнении условия (1) левая часть последнего равенства больше нуля, ибо ее можно представить в виде

$$
\begin{aligned}
\int_{E_{1}}\left(b_{0} Q_{0}(x)+b_{1} Q_{1}(x)+\cdots+b_{n-1} Q_{n-1}(x)\right)^{2} \rho_{+}(x) d x \\
\quad+\int_{E_{2}}\left(b_{0} Q_{0}(x)+b_{1} Q_{1}(x)+\cdots+b_{n-1} Q_{n-1}(x)\right)^{2} \rho_{-}(x) d x .
\end{aligned}
$$


Отметим, что по аналогии с рядом Фурье по системе (4) можно определить ряд Фурье по системе (12) для любой $f \in L_{2, \rho}(a, b)$ следующим образом

$$
(f, \rho)(x) \sim \sum_{n=0}^{\infty} c_{n}\left(Q_{n}, \rho\right)(x),
$$

где

$$
\begin{aligned}
& c_{0}=c_{0}(f)=\int_{a}^{b}(f, \rho)(x) Q_{0}(x) d x \\
& c_{n}=c_{n}(f)=\int_{a}^{b}(f, \rho)(x) Q_{n}(x) d x-\sum_{k=0}^{n-1} c_{k} \int_{a}^{b}\left(Q_{k}, \rho\right)(x) Q_{n}(x) d x,
\end{aligned}
$$

$n=1,2, \ldots$.

Систему (12) ниже будем назьвать сопряженной (с системой (4)) несимметрично ортонормированной системой полиномов.

Переходя к аналогам свойств нулей ортогональных полиномов, заметим, что каждый полином $P_{n}(x)(n=1,2, \ldots)$ из системы (4) имеет хотя бы один нуль на $(a, b)$. Это следует из равенства

$$
\int_{a}^{b} P_{n}(x)\left(P_{0}, \rho\right)(x) d x=0 \quad(n=1,2, \ldots) .
$$

Для сопряженной системы (12) имеет место утверждение, вполне аналогичное свойству нулей ортогональных полиномов.

ТЕОРема 3. Если вес $\rho(x)=\left(\rho_{-}(x), \rho_{+}(x)\right)$ удовлетворяет условию (1), то каждый полином $Q_{n}(x)(n=1,2, \ldots)$ из сопряженной несимметрично ортонормированной системы (12) имеет $n$ различных нулей на интервале $(a, b)$.

ДоКАЗАТЕЛЬСТво. Сначала покажем, что каждьй полином $Q_{n}(x)$ $(n=1,2, \ldots)$ имеет хотя бы один нуль на $(a, b)$.

Действительно, пусть, например, $Q_{n}(x)>0, x \in(a, b)$. Тогда из (1) имеем

$$
\int_{a}^{b}\left(Q_{n}, \rho\right)(x) d x \geqslant \int_{a}^{b} Q_{n}(x) m(x, \rho) d x>0,
$$

что противоречит равенству $\left(Q_{0}, Q_{n}\right)_{\rho}=0$.

Пусть $Q_{n}(x)$ имеет $k$ нулей $x_{1}, x_{2}, \ldots, x_{k}$ на $(a, b)$, и допустим, что $k<n$. Тогда, представив полином $Q(x)=\left(x-x_{1}\right)\left(x-x_{2}\right) \cdots\left(x-x_{k}\right)$ в виде $Q(x)=c_{0} Q_{0}(x)+c_{1} Q_{1}(x)+\cdots+c_{k} Q_{k}(x)$, по свойству полиномов $Q_{k}(x)$ получим

$$
J_{1}=\int_{a}^{b} Q(x)\left(Q_{n}, \rho\right)(x) d x=0 .
$$


С другой стороны, этот же интеграл равен

$$
J_{1}=\int_{a}^{b} Q(x) Q_{n}(x) \rho\left(x, Q_{n}\right) d x
$$

где $\rho\left(x, Q_{n}\right)=\rho_{+}(x) \operatorname{sign} Q_{n}^{+}(x)+\rho_{-}(x) \operatorname{sign} Q_{n}^{-}(x), \quad$ а произведение $Q(x) Q_{n}(x)$ сохраняет свой знак на $(a, b)$, причем, используя свойство $(1)$ веса $\rho(x)$, имеем: если $Q(x) Q_{n}(x) \geqslant 0$, то

$$
J_{1} \geqslant \int_{a}^{b} Q(x) Q_{n}(x) m(x, \rho) d x>0
$$

если же $Q(x) Q_{n}(x) \leqslant 0$, то $J_{1}<0$.

Следовательно, $k=n$.

Если допустить, что $Q_{n}(x)$ имеет кратньй нуль $x_{0}$, то, рассмотрев полином $R(x)=Q_{n}(x)\left(x-x_{0}\right)^{-2}$ степени $n-2$, получим

$$
J_{2}=\int_{a}^{b} R(x)\left(Q_{n}, \rho\right)(x) d x=0 .
$$

С другой стороны,

$$
\begin{aligned}
J_{2} & =\int_{a}^{b} Q_{n}^{2}(x)\left(x-x_{0}\right)^{-2} \rho\left(x, Q_{n}\right) d x \\
& \geqslant \int_{a}^{b} Q_{n}^{2}(x)\left(x-x_{0}\right)^{-2} m(x, \rho) d x>0 .
\end{aligned}
$$

Как приложение теоремы 3 получим аналог классической квадратурной формулы Гаусса.

ТЕОРема 4. Пусть $Q_{0}(x), Q_{1}(x), \ldots$ - сопрязсенная несимметрично ортонормированная система полиномов с весом $\rho(x)=\left(\rho_{-}(x)\right.$, $\left.\rho_{+}(x)\right)$ на интервале $(a, b)$ и пусть $x_{1}<x_{2}<\cdots<x_{n}-$ нули полинома $Q_{n}(x)$ для данного $n=1,2, \ldots$. Тогда существуют неотрииательные числа $\lambda_{1}, \lambda_{2}, \ldots, \lambda_{n}$ такие, что для любого полинома $Q(x)$ степени не выше $2 n-1$ имеет место равенство

$$
\int_{a}^{b} Q(x) \rho\left(x, Q_{n}\right) d x=\sum_{k=1}^{n} \lambda_{k} Q\left(x_{k}\right)
$$

əде $\rho\left(x, Q_{n}\right)=\rho_{+}(x) \operatorname{sign} Q_{n}^{+}(x)+\rho_{-}(x) \operatorname{sign} Q_{n}^{-}(x)$. 
ДоКАЗАТЕЛЬСТво аналогично доказательству теоремы 3.4.1 из [5]. Укажем лишь значения коэффициентов:

$$
\lambda_{k}=\int_{a}^{b} \frac{\left(Q_{n}, \rho\right)(x)}{Q_{n}^{\prime}\left(x_{k}\right)\left(x-x_{k}\right)} d x .
$$

Из (13) при $k=1,2, \ldots, n$ непосредственно следует, что

$$
\lambda_{k}=\int_{a}^{b}\left[\frac{Q_{n}(x)}{Q_{n}^{\prime}\left(x_{k}\right)\left(x-x_{k}\right)}\right]^{2} \rho\left(x, Q_{n}\right) d x,
$$

а следовательно, $\lambda_{k} \geqslant 0, k=1,2, \ldots, n$.

Далее, если числа $x_{k}$ и $\lambda_{k}(k=1,2, \ldots, n)$ те же, что и в теореме 4 , то для любой, например, непрерьвной на $[a, b]$ функции $f(x)$ имеем

$$
\int_{a}^{b} f(x) \rho\left(x, Q_{n}\right) d x=\sum_{k=1}^{n} \lambda_{k} f\left(x_{k}\right)+R(f),
$$

причем остаточный член $R(f)$ можно найти, используя стандартные рассуждения, если $f(x)$ является $2 n$ раз дифференцируемой на $[a, b]$; в этом случае для $\omega(x)=\left(x-x_{1}\right) \cdots\left(x-x_{n}\right)$ и некоторой точки $t \in(a, b)$ вьполняется равенство

$$
R(f)=\frac{1}{(2 n) !} f^{(2 n)}(t) \int_{a}^{b} \omega^{2}(x) \rho\left(x, Q_{n}\right) d x .
$$

Дагестанский государственный университет

Поступило

29.12 .94

\section{СПИСОК ЦИТИРОВАННОЙ ЛИТЕРАТУРЫ}

[1] Долженко Е.П., Севастьянов Е. А.Знакочувствительные аппроксимации. Пространство знакочувствительных весов. Жесткость и свобода системы // Докл. РАН. 1993. Т. 332. №6. С. 686-689.

[2] Долженко Е. П., Севастьянов Е. А. Знакочувствительные аппроксимации. Вопросы единственности и устойчивости // Докл. РАН. 1993. Т. 333. № 1. C. $5-7$.

[3] Бабенко В.Ф. Несимметричные приближения в пространствах суммируемых функций // УМН. 1982. Т. 34. №4. С. 409-420.

[4] Суетин П. К. Классические ортогональные полиномы. М.: Наука, 1976.

[5] Сегё Г. Ортогонаљные многочлены. М.: Физматгиз, 1962.

[6] Натансон И.П. Теория функций вещественной переменной. М.: Наука, 1974. 